\title{
Photocatalytic degradation activity of goji berry extract-mediated green synthesized antibacterial silver-zinc oxide nanocomposites under simulated solar light irradiation
}

\author{
Abdulrahman Ahmed Sharwani \\ Yeungnam University \\ Kannan Badri Narayanan \\ Yeungnam University \\ Mohammad Ehtisham Khan \\ Jazan University \\ Sung Soo Han ( $\square$ sshan@yu.ac.kr ) \\ Yeungnam University
}

\section{Research Article}

Keywords: silver, zinc oxide, goji berry extract, nanocomposite, photocatalysis, antimicrobial

Posted Date: March 3rd, 2022

DOI: https://doi.org/10.21203/rs.3.rs-1395775/v1

License: (c) (1) This work is licensed under a Creative Commons Attribution 4.0 International License.

Read Full License 


\section{Abstract}

Green synthesis has gained extensive attention in material science because of its sustainability and ecofriendliness in producing a wide range of nanomaterials. This study demonstrated the photocatalytic and antimicrobial abilities of green synthesized silver-embedded zinc oxide nanocomposites (Ag@ZnO NCs). Zinc oxide with a unique mesoporous ellipsoidal morphology in size range of $0.59 \pm 0.11 \times 0.33 \pm 0.09$ $\mu \mathrm{m}$ (length $\times$ width) was synthesized using the aqueous extract of goji berry ( $\mathrm{ZnO}-\mathrm{GB}$ ) by calcination in the air. Powder X-ray diffraction (XRD) revealed the formation of a hexagonal phase of wurtzite (WZ) structure. The average crystallite size of ZnO-GB was $23.74 \pm 4.9 \mathrm{~nm}$ as calculated using DebyeScherrer's equation. It also possesses higher thermal stability with the surface area, pore-volume, and pore size of $11.77 \mathrm{~m}^{2} / \mathrm{g}, 0.027 \mathrm{~cm}^{3} / \mathrm{g}$, and $9.52 \mathrm{~nm}$, respectively. Furthermore, different mesoporous Ag@ZnO NCs embedded with face-centered cubic (fcc) silver nanoparticles (Ag NPs) in the range of 90$160 \mathrm{~nm}$ were synthesized by GB extract as a reducing and capping agent on the surface of ZnO-GB after calcination. The immobilization of Ag NPs was confirmed by XRD, X-ray photoelectron spectroscopy (XPS), field-emission scanning electron microscopy (FE-SEM), FE-transmission electron microscopy (FETEM), and energy-dispersive X-ray spectroscopy (EDS). It was found that $\mathrm{Ag}_{0.2} @ \mathrm{ZnO} \mathrm{NC}$ (0.2 wt\% of Ag) showed excellent photocatalytic degradation of both methylene blue (MB) (cationic) and congo red (CR) (anionic) dyes under simulated solar irradiation. The photocatalytic degradation of $99.3 \pm 0.35 \% \mathrm{MB}$ and $98.5 \pm 1.3 \%$ CR occurred in 90 and 55 min, respectively, at room temperature by Ag $_{0.2} @ Z n O N C$. Besides, these NCs also showed broad-spectrum antibacterial activity against both Gram-positive and Gramnegative bacteria. The mechanistic concept of generating reactive oxygen species (ROS) by electron and hole charge $\left(\mathrm{e} / / \mathrm{h}^{+}\right)$carriers seems to be responsible for the photocatalytic degradation of commercial dyes and antibacterial activities by Ag@ZnO NCs. Thus, these as-prepared green Ag@ZnO NCs are promising candidates as photocatalysts for industrial/wastewater treatment as well as in antimicrobial therapeutics.

\section{Introduction}

Advanced oxidation processes (AOPs) are widely used technology to remove any organic recalcitrant contaminants/pollutants in wastewater. Generally, AOPs such as Fenton-like processes, ozonation, sonolysis, and photocatalysis are used for the remediation treatments in the aqueous medium ${ }^{1,2}$. These AOPs utilize strong oxidants such as hydroxyl and superoxide anion radicals for the remediation of pollutants and antimicrobial activity. However, the drawbacks of AOPs are mainly because of their expensive energy sources such as ultraviolet (UV) light or reagents such as ozone and hydrogen peroxide. Thus, the use of solar irradiation as a natural energy source in photocatalysis can reduce the cost of remediation. Photocatalysis is a cost-effective, eco-friendly, and sustainable catalytic process involving light energy as a renewable energy source to activate photocatalysts such as metal or semiconductor nanoparticles to degrade various environmental hazardous pollutants ${ }^{3}$. It is a surface phenomenon that happens mainly on the catalyst's surface. The photocatalytic efficiency of any nanomaterials is based on

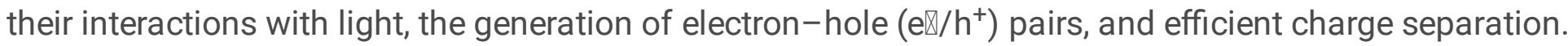


In the recent past, researchers have used several photocatalysts to degrade several hazardous industrial dyes and pollutants. The dyes are classified into cationic and anionic dyes that can be dissociated into positively and negatively charged ions, respectively, in an aqueous solution ${ }^{4}$. The cationic dyes such as methylene blue (MB), rhodamine $B(\mathrm{Rhb})$, crystal violet (CV), rhodamine $6 \mathrm{G}(\mathrm{Rh} 6 \mathrm{G})$, and malachite green $(M G)$, and anionic dyes such as methyl orange (MO), acid orange 7 (AO7), phenol red (PR), eosin $Y$ (EY), congo red (CR), and rose Bengal (RB) are widely used in textile industries and discharged as industrial effluents ${ }^{5,6}$. Other organic pollutants include glyphosate, carbofuran, picloram, fluometuron, aniline, methamodiphos, trichlorfon, turbophos, trichlopyr, erioglaucine, tebuthioron, and propham ${ }^{7}$. Using photocatalysis, both dyes and other organic pollutants can be mineralized to carbon dioxide and water without any secondary hazardous products ${ }^{8,9}$.

Metal and semiconductor nanoparticles exhibit unique optoelectronic properties depending on their size and shape for the photoinduced catalytic reactions ${ }^{10}$. Semiconductors such as zinc oxide ( $\mathrm{ZnO}$ ) and titania $\left(\mathrm{TiO}_{2}\right)$ can act as photocatalysts. Among different semiconductors, $\mathrm{ZnO}$ is one of the most common biocompatible transition metal oxide semiconductors of the II-VI semiconductor group used. The low photocatalytic activity of bulk $\mathrm{ZnO}$ is due to its quick recombination of charge carriers and comparatively low charge separation. However, ZnO nanoparticles (NPs) with hexagonal wurtzite crystal structure exhibit good photoexcitation stability and high electron mobility. Besides, the nanostructured $\mathrm{ZnO}$ with a wide bandgap of $3.37 \mathrm{eV}$ cannot show any absorption upon visible light irradiation ${ }^{11}$. Therefore, the doping of noble metals, metal oxides, metal sulfides, or polymers in the preparation of semiconductor nanocomposites can enhance the absorption in the visible range ${ }^{12}$. In addition, the synthesis of nanomaterials with metal nanoparticles of silver, gold, iron, copper, ruthenium, and palladium on the surfaces of metallic oxides has significant applications in the diverse fields of biosensing, photovoltaics, energy storage, and optics along with catalysis ${ }^{13}$. For instance, semiconductor metal oxide nanoparticles of ZnO NPs exhibit promising applications in photocatalysis, heterogeneous catalysis, and antimicrobial therapy ${ }^{14,15}$. The UV light-driven photocatalytic activity of ZnO NPs is due to their wide bandgap and high exciton binding energy $(60 \mathrm{meV})$. It is also known to generate reactive oxygen species (ROS), which enhances photocatalytic activity and biomedical applications for antibacterial and photodynamic therapy ${ }^{16}$. The extent of ROS production and the cytotoxicity of ZnO NPs are enhanced by the interaction with cellular components and the release of Zinc cations. Similarly, metal nanoparticles such as silver nanoparticles (Ag NPs) can induce oxidative stress and cellular toxicity by producing ROS species. The surface plasmon resonance (SPR) of Ag NPs can give visible light photocatalysis to $\mathrm{Ag} / \mathrm{ZnO}$ nanocomposites. The combination of $\mathrm{ZnO}$ and $\mathrm{Ag}$ together can generate more ROS and provide a cumulative effect on photocatalysis, antibacterial and anticancer activities ${ }^{17}$.

Green synthesis of nanocomposites is emerging as an eco-friendly sustainable methodology for the lowcost synthesis of various metal and semiconductor nanocomposites ${ }^{18}$. Demissie, et al. ${ }^{14}$ synthesized ZnO NPs using Lippia adoensis "Koseret" leaf extract and evaluated their antibacterial activity against both Gram-positive (Staphylococcus aureus and Enterococcus faecalis) and Gram-negative (Escherichia 
coli and Klebsiella pneumonia) bacteria. Similarly, using the aqueous extract of wolfberry fruit extract, Dong and colleagues green synthesized highly crystalline spherical Ag NPs in the range between 3 and $15 \mathrm{~nm}{ }^{19}$. Recently, Chauhan, et al. ${ }^{20}$ employed a facile green route to synthesize both $\mathrm{ZnO}$ and Ag-doped $\mathrm{ZnO}$ using the leaf extract of Cannabis sativa as a reducing and stabilizing agent. These nanoparticles were demonstrated for the photocatalytic degradation of industrial dyes (congo red and methyl orange) and antimicrobial activity. Different morphologies of nanomaterials can be synthesized in a greener route by controlling the synthesis parameters. Herein, we have developed a green methodology to synthesize unique mesoporous ellipsoidal semiconductor particles of zinc oxide (ZnO-GB) and metal/semiconductor nanocomposites of silver@zinc oxide nanocomposites (Ag@ZnO NCs) using the aqueous fruit extract of goji berries (GB) as a reducing and stabilizing agent. ZnO-GB and Ag@ZnO NCs with different silver concentrations have been evaluated for their photocatalytic activity against both anionic and cationic dyes and antibacterial activity against Gram-positive (S. aureus) and -negative (E. coli) bacteria.

\section{Experimental}

\section{Materials}

Silver nitrate $\left(\mathrm{AgNO}_{3}, 99 \%\right)$ was purchased from Sigma-Aldrich (USA). Zinc nitrate hexahydrate (extra pure grade), ammonia water, methylene blue (MB), and congo red (CR) were purchased from Duksan Pure Chemicals Co., Ltd. (South Korea). Ampicillin sodium salt was bought from Daejung Chemicals \& Metals Co., Ltd. (Siheung, South Korea). Dried goji berries (Lycium barbarum L.) were purchased from Yeongcheon medicinal herb market (Yeongcheon, South Korea). Microorganisms Escherichia coli (KCTC 2571) and Staphylococcus aureus (KCTC 3881) were obtained from the Korean Collection for Type Cultures (Jeongeup, South Korea). Muller-Hinton $(\mathrm{MH})$ broth medium and agar were purchased from Becton, Dickinson, and Company (Sparks, MD, USA). Deionized water was collected using a Milli-Q direct water purification system (Merck Millipore) and used to prepare all solutions.

\section{Preparation of goji berry (GB) extract}

The aqueous extract of goji berry (GB) (Lycium barbarum L.) fruit was done as mentioned earlier ${ }^{21}$. Briefly, the dried GBs were chopped into small pieces and then excellently ground into a coarse powder in a mortar pestle. The aqueous extract was prepared by heating $5.0 \mathrm{~g}$ of GB powder in $100 \mathrm{~mL}$ of deionized water taken in a $250 \mathrm{~mL}$ Erlenmeyer flask and allowed to boil with stirring at $100{ }^{\circ} \mathrm{C}$ for $15 \mathrm{~min}$. Later, the solution was cooled to room temperature and centrifuged at 4,000 rpm for $10 \mathrm{~min}$ and filtered through Whatman No. 1 filter paper to obtain a clarified solution of GB extract. Finally, the aqueous GB extract was stored in the refrigerator at $4{ }^{\circ} \mathrm{C}$ for the preparation of metal nanoparticles and metal/semiconductor nanocomposites.

\section{Goji berry-mediated synthesis of zinc oxide particles (ZnO-GB)}

Initially, $8.0 \mathrm{~g}$ zinc nitrate hexahydrate was dissolved in $100 \mathrm{~mL}$ deionized water and stirred at room temperature for $5 \mathrm{~min}$. Then, $30 \mathrm{~mL}$ of freshly prepared GB extract solution was dropwise added, and the 
$\mathrm{pH}$ of the solution was adjusted to 9.0 using aqueous ammonia. The resultant mixture was continuously stirred for $24 \mathrm{~h}$ at $80^{\circ} \mathrm{C}$. The obtained yellow precipitate was collected by centrifugation at 4,000 rpm for $15 \mathrm{~min}$ and washed twice with deionized water. The as-prepared GB-mediated zinc oxide (ZnO-GB) particles were dried in a vacuum oven at $60^{\circ} \mathrm{C}$ overnight, followed by calcination at $200{ }^{\circ} \mathrm{C} / 2 \mathrm{~h}$ and 250 ${ }^{\circ} \mathrm{C} / 3 \mathrm{~h}$ in air and stored in an airtight amber vial.

\section{Preparation of silver@zinc oxide nanocomposites (Ag@ZnO NCs)}

To prepare different Ag@ZnO NCs, 6.0 g ZnO-GB was added with varying quantities of silver $(0.2 \%, 0.4 \%$, and $0.8 \%$ ) in $100 \mathrm{~mL}$ of deionized water taken in an amber bottle. The solution was sonicated for $30 \mathrm{~min}$ to homogeneous the solution containing silver nitrate and ZnO-GB. Then $40 \mathrm{~mL}$ GB extract was added dropwise with constant stirring at $60^{\circ} \mathrm{C}$ for $3 \mathrm{~h}$. The formed precipitate was washed three times with deionized water after centrifuging at 10,000 rpm for $20 \mathrm{~min}$ and dried in a hot air oven at $60^{\circ} \mathrm{C}$ overnight. These dried nanocomposite powders were calcined in air at $200{ }^{\circ} \mathrm{C} / 2 \mathrm{~h}$ and $250{ }^{\circ} \mathrm{C} / 3 \mathrm{~h}$ and stored in an amber vial for further experiments. These nanocomposites with different silver concentrations of $0.2,0.4$, and 0.8\% (wt/v) were referred to as $\mathrm{Ag}_{0.2} @ \mathrm{ZnO}, \mathrm{Ag}_{0.4} @ \mathrm{ZnO}$, and $\mathrm{Ag}_{0.8} @ \mathrm{ZnO} \mathrm{NCs}$, respectively. The pictorial representation of the synthesis of these metal/semiconductor nanocomposites is provided as the schematic diagram (Figure 1).

\section{Characterization of ZnO-GB and Ag@ZnO NCs}

The optoelectronic properties of ZnO-GB and different Ag@ZnO NCs were determined from the ultravioletvisible (UV-vis) diffuse reflectance spectra (DRS) recorded using a VARIAN Cary 5000 spectrophotometer (Agilent Technologies, USA) equipped with a Praying Mantis diffuse reflectance accessory (DRA). Powder X-ray diffraction (XRD) analysis was performed to determine the crystalline structure of the nanocomposites using a PANalytical X'PertPRO MPD (Netherlands) X-ray diffractometer with Cu Ka1 radiation $(0.15406 \mathrm{~nm})$ and operating conditions of tube voltage $40 \mathrm{kV}$, tube current $30 \mathrm{~mA}$, and scanning between $7.0^{\circ}$ and $80.0^{\circ}(2 \theta)$ at a rate of $1.2^{\circ} / \mathrm{min}$. The diffraction peaks of the crystalline phases were compared with the standard compounds of the JCPDS data. The average crystallite size (D) of the samples was calculated using the Debye-Scherrer's equation: $D=K \lambda / \beta \cos \theta$, where $K$ is Debye-Scherrer constant (0.89), $\lambda$ is the X-ray wavelength $(0.15406 \mathrm{~nm}), \beta$ is the full-width at half maximum (FWHM), and $\theta$ is the diffraction angle. Fourier-transform infrared (FTIR) spectroscopy was performed using a PerkinElmer FTIR (Model: Spectrum 100) spectrometer in transmittance mode with the wavenumber range of $400-4000 \mathrm{~cm}^{-1}$. The hydrodynamic size and zeta potential of the samples were analyzed using Zetasizer nanoparticle analyzer (Malvern Instruments Worc, UK, Model: ZS90) at $25^{\circ} \mathrm{C}^{22}$.

To analyze the morphology and composition of the nanocomposites, field-emission scanning electron microscopy (FE-SEM) (Hitachi, Japan, Model: S-4200) was performed by mounting the samples on an aluminum stub and sputter-coated with platinum and analyzed with secondary electron (SE) detectors at operating voltages of 10 and $15 \mathrm{kV}$ and the elemental composition was analyzed by SEM-energy dispersive X-ray spectroscopy (SEM-EDX). The shape and size of the nanocomposites were examined 
using an FE-transmission electron microscope (FE-TEM, FEI Tecnai G2 F20, Oregon, USA) at an accelerating voltage of $200 \mathrm{kV}$. The elemental analysis of the nanocomposites was also analyzed using the high-angle annular dark-field scanning TEM energy-dispersive X-ray spectroscopy (HAADF-STEMEDS). The oxidation state of each element of the nanocomposite was analyzed using X-ray photoelectron spectroscopy (XPS) via a Thermo Scientific K-Alpha system with an Al Ka X-ray source and the ion source energy was between $100 \mathrm{~V}$ and $3.0 \mathrm{keV}$ for the survey ${ }^{21}$. The thermal stability of nanocomposites was analyzed by thermogravimetric analysis (TGA) from room temperature to $800^{\circ} \mathrm{C}$ at a heating rate of 20 ${ }^{\circ} \mathrm{C} / \mathrm{min}$ in a nitrogen atmosphere. Photoluminescence $(\mathrm{PL})$ spectroscopy was performed using the HORIBA Scientific Raman system and analyzed with LabSpec 6 software. A $325 \mathrm{~nm}$ air-cooled He-Cd laser power at $50 \mathrm{~mW}$ with Syncerity CCD and detected with 10x objective in the wavelength range of 340-1050 nm. Brunauer-Emmett-Teller (BET) surface area $\left(S_{B E T}\right)$, Barrett-Joyner-Halenda $(B J H)$ pore size distribution, and pore-volume of samples were analyzed using a Micromeritics 3Flex adsorption analyzer (Norcross, GA, USA). The photocatalytic degradation of dyes was evaluated using a Shimadzu UV-2600 dual-beam UV-vis spectrophotometer (Kyoto, Japan).

\section{Applications of ZnO-GB and Ag@ZnO NCs}

\section{Photocatalytic degradation of dyes}

The photocatalytic degradation of dyes (MB and CR) by ZnO-GB and Ag@ZnO NCs as photocatalysts was assessed by the decolorization of dye solutions with the initial concentrations of $10 \mathrm{mg} / \mathrm{L} \mathrm{MB}$ or 20 $\mathrm{mg} / \mathrm{L} \mathrm{CR}$ under simulated solar light irradiation (Osram Ultra-Vitalux, $300 \mathrm{~W}$ ). In the photocatalysis, $0.1 \%$ (w/v) of ZnO-GB and various Ag@ZnO NCs were taken as photocatalysts and added to $100 \mathrm{ml}$ of aqueous dye solutions under continuous stirring. Before simulated solar irradiation, the dye solution with photocatalyst was incubated at room temperature in the dark for 30 min to reach adsorption-desorption equilibrium. The distance between the lamp and the dye solution was kept at $10 \mathrm{~cm}$, and the samples were taken periodically and centrifuged at $12,000 \mathrm{rpm}$ for $10 \mathrm{~min}$ to remove the nanocomposites from the dye solutions. The maximum absorbance $\left(\lambda_{\max }\right)$ of the supernatant dye solution was analyzed by a dualbeam UV-vis spectrophotometer to quantify the concentrations of MB and CR dyes at 663 and $498 \mathrm{~nm}$, respectively. The rate of degradation of dyes was calculated by the percentage of the concentration of dye that remained after a specific time over the initial dye concentration.

Degradation $(\%)=\left(C_{0}-C_{t} / C_{0}\right) \times 100$

Where $\mathrm{C}_{0}$ and $\mathrm{C}_{\mathrm{t}}$ are the initial and final concentrations of dyes at a reaction time (t), respectively.

\section{Antibacterial assay}

The antibacterial activity of ZnO-GB and Ag@ZnO NCs was tested against both Gram-negative (E. coli) and -positive (S. aureus) bacteria using the agar well diffusion method ${ }^{23}$. The overnight cultures of $E$. coli and $S$. aureus was obtained by inoculating the $\mathrm{MH}$ broth with the pure single colonies of bacteria. Later, the $\mathrm{MH}$ agar plates were spread-plated with pure bacterial suspensions, and the agar wells were made 
using a sterile cork-borer with a diameter of 8 mm. Different Ag@ZnO NCs and ZnO-GB (2 mg, 40 mg/ml) were loaded into the wells, and the plates were incubated at $37^{\circ} \mathrm{C}$ for $16 \mathrm{~h}$. Ampicillin $(300 \mu \mathrm{g}$ for $S$. aureus, and $500 \mu \mathrm{g}$ for $E$. coli) was used as a positive control. The development of the zone of inhibitions (ZOIs) around the ZnO-GB and Ag@ZnO NCs loaded wells was measured and recorded.

\section{Results And Discussion}

\section{Green synthesis of ZnO-GB and Ag@ZnO NCs}

Generally, plant extracts have great potential in the synthesis of nanoparticles and nanocomposites. The aqueous extract of goji berries contains several phytochemicals such as phenylpropanoids, coumarins, lignans, and isoflavonoids providing natural reduction, capping, and/or stabilization moieties over the expensive chemicals to form metal nanoparticles and nanocomposites ${ }^{21}$. During the green synthesis of $\mathrm{ZnO}-\mathrm{GB}$, the color of the solution changed to light yellowish and precipitated within $30 \mathrm{~min}$ at $80^{\circ} \mathrm{C}$, indicating the formation of ZnO-GB. The possible mechanism involved in the formation of $\mathrm{ZnO}-\mathrm{GB}$ particles could be that the polyphenols in the GB extract could have acted as ligand agents forming complex ligands with zinc ions, and the stabilized ZnO-GB particles were formed through the process of nucleation and condensation. The excess of bioorganic components attached to $\mathrm{ZnO}-\mathrm{GB}$ from the $\mathrm{GB}$ extract could have been decomposed in the calcination process in air releasing $\mathrm{ZnO}-\mathrm{GB}$ particles ${ }^{24,25}$. Sadiq, et al. ${ }^{15}$ demonstrated the green synthesis of ZnO NPs using the leaf extract of Syzygium cumini (black plum). Besides secondary metabolites, plant extracts also contain many biomolecules such as proteins, polysaccharides, terpenoids, and alkaloids that could have been involved in the bioreduction and stabilization of various metal/metal oxide nanoparticles and nanocomposites ${ }^{26}$. When different concentrations of silver (0.2-0.8 wt\%) were mixed with ZnO-GB and with the addition of GB extract, the solution color changed to light greenish, denoting the formation of Ag NPs on the surface of ZnO-GB as Ag@ZnO NCs at $60^{\circ} \mathrm{C}$ within $3 \mathrm{~h}$ (Figure 1). The optical properties of the colloidal solution depend on the nanoscale morphology as well as the distance between them ${ }^{27}$. It has been postulated that the keto-enol tautomeric transformation of polyphenolic compounds of plant extract such as flavonoids may release the reactive hydrogen atoms, which drive the reduction of $\mathrm{Ag}$ ions and enable the formation of $\mathrm{Ag} \mathrm{NPs}{ }^{28-}$ 30. In addition, the internal conversion of ketones to carboxylic acids in flavonoids was also likely to be involved in the reduction process of silver ions to $\mathrm{Ag} \mathrm{NPs}^{31}$.

\section{Characterization of ZnO-GB and Ag@Zno NCs}

The optical properties of the ZnO-GB and Ag@ZnO NCs were investigated by the UV-vis diffuse reflectance absorption spectra. Figure 2a shows the absorption edges of ZnO-GB and Ag@ZnO NCs in the UV region with a band edge at $~ 372 \mathrm{~nm}$. However, Ag@ZnO NCs with Ag NPs showed better absorption in the visible region from 450 to $550 \mathrm{~nm}$, and the intensity of the absorption in the visible region is solely dependent on the absorption of Ag NPs ${ }^{32}$. The broad bands of NCs in the visible region are mainly due to the surface plasmon resonance (SPR) of Ag NPs indicating the polydispersity nature of nanoparticles. 
The increase in the plasmon peak intensity is correlated with the increase in the average size of the $\mathrm{Ag}$ NPs, and the absorption band for Ag NPs shifted towards a higher wavelength with the increasing Ag content. The bandgap energies $(E g)$ of all samples were calculated using the following equation: $a h v=$ $A\left(h v-E_{g}\right)^{n}$, where $E g$ is the direct bandgap energy, $a$ is the optical absorption coefficient, $h v$ is the photon energy, $n$ corresponds to the nature of transition, and $A$ is the constant. The bandgap energies of all samples were calculated from the Tauc's plot, and the bandgap diagram and values are shown in Figure 2b. The optical bandgap energy of ZnO-GB was $3.1 \mathrm{eV}$, and the bandgap values of Ag@ZnO NCs decreased with increased Ag NPs binding on the surface of ZnO. The bandgap values were 2.99, 2.93, and 2.88 eV for Ag $_{0.2} @ Z n 0, A_{0.4} @ Z n 0$, and Ag ${ }_{0.8} @ Z n O N C s$, respectively. The lower bandgap energies of Ag@ZnO NCs were attributed to the introduction of impurity into the ZnO grains that have trapped excited electrons from the conduction band and promoted continuum of energy level and bandgap narrowing ${ }^{33}$. Besides, the GB extract components modify/stabilize the surface of NPs and NCs and reduce the bandgap values ${ }^{34}$.

The purity and crystal structure of ZnO-GB and Ag@ZnO NCs, synthesized by the GB extract, were determined through powder XRD spectra, as shown in Figure 2c. Powder XRD spectra revealed the characteristic $2 \theta$ peaks of $\mathrm{ZnO}-\mathrm{GB}$ at $31.76^{\circ}, 34.43^{\circ}, 36.26^{\circ}, 47.56^{\circ}, 56.61^{\circ}, 62.90^{\circ}, 66.41^{\circ}, 67.98^{\circ}, 69.10^{\circ}$, $72.61^{\circ}, 77.0^{\circ}$ corresponding for (100), (002), (101), (102), (110), (103), (200), (112), (201), (004), and (202) planes of the crystal lattices. This agrees with the polycrystalline diffraction patterns of the hexagonal phase of wurtzite ZnO (JCPDS card No. 89-0510), and no impurity peaks were observed ${ }^{35}$. Qu, et al. ${ }^{36}$ demonstrated the synthesis of ZnO NPs with hexagonal wurtzite crystal structure from the Sedum alfredii Hance, a Zinc hyperaccumulating plant. In another instance, a bio-based approach was used to synthesize crystalline and polydispersed ZnO NPs $(72.5 \mathrm{~nm})$ using Physalis alkekengi L. to remediation of zinc-contaminated soils ${ }^{37}$. The average crystallite size of ZnO-GB calculated using the DebyeScherrer's equation was $23.74 \pm 4.9 \mathrm{~nm}$. Also, the strong and sharp diffraction peaks confirm the high crystallinity of $\mathrm{ZnO}-\mathrm{GB}$, and the degree of crystallinity was calculated through the equation: [area of crystalline peaks/area of crystalline \& amorphous peaks] $\times 100$, showed $100 \%$ crystallinity. Zaid, et al. ${ }^{38}$ reported that calcination at higher temperatures could improve the crystallinity and better particle distribution. In Ag@ZnO NCs, the additional peaks of $38.11^{\circ}, 44.30^{\circ}$, and $64.45^{\circ}$ correspond to (111), (200), and (220) planes of face-centered cubic (fcc) phase of silver (JCPDS card No. 2-109) ${ }^{39}$. The ionic radius of silver ion $\left(\mathrm{Ag}^{+}\right)(0.122 \mathrm{~nm})$ was larger than that of zinc divalent $\left(\mathrm{Zn}^{2+}\right)$ ions, thus silver ions cannot be substituted into the crystal lattice of the $\mathrm{ZnO}$ matrix, therefore, the metallic silver peaks due to the Ag NPs are formed over the ZnO surface ${ }^{33}$. The FWHM and crystallite size are inversely proportional, therefore, the increase in the size of Ag NPs results in the formation of larger NCs. These Ag NPs formed on the surface of ZnO-GB were in the size of $25.65 \pm 5.0,32.91 \pm 3.3$, and $33.32 \pm 4.21 \mathrm{~nm}$ diameter for Ag ${ }_{0.2} @ Z n O, A g_{0.4} @ Z n O$, and Ag $0.8 @ Z n O N C s$, respectively. The intensity of Ag NPs peaks increases with the increase in the silver content of NCs, which is due to the increase in the number of Ag NPs on the surface of $\mathrm{ZnO}$. 
The functional groups involved in the formation of ZnO-GB and Ag@ZnO NCs were investigated by the Fourier-transform infrared (FTIR) spectroscopy in the range of $400-4000 \mathrm{~cm}^{-1}$ (Figure 2d). FTIR spectra of all samples and GB extract exhibited various absorption bands. In GB extract spectrum, the broad band centered at $3290 \mathrm{~cm}^{-1}$ was assigned to hydrogen-bonded $\mathrm{O}-\mathrm{H}$ stretching vibrations, and the weak signal at $2936 \mathrm{~cm}^{-1}$ was due to $\mathrm{C}-\mathrm{H}$ stretching vibrations ${ }^{40}$. The band at $1595 \mathrm{~cm}^{-1}$ was attributed to the $\mathrm{C}-$ $\mathrm{OH}$ deformation vibration, and the band at $1417 \mathrm{~cm}^{-1}$ was due to the $\mathrm{O}-\mathrm{C}-\mathrm{O}$ symmetric and asymmetric stretching vibrations of the carboxylate group. Moreover, the band at $1025 \mathrm{~cm}^{-1}$ was assigned to $\mathrm{C}-0$ stretching vibrations of the pyranose ring ${ }^{21,41}$. The FTIR spectra of GB extract synthesized ZnO-GB and Ag@ZnO NCs exhibited a difference from the GB extract spectrum. The intensity of broad band around wavenumber $3396 \mathrm{~cm}^{-1}$, the characteristic of $\mathrm{OH}$ stretching vibration, decreased in all samples after calcination ${ }^{42}$. Meanwhile, the broad absorption bands around $400-600 \mathrm{~cm}^{-1}$ were attributed to the stretching modes of metal-oxygen bonds, thus confirming the formation of $\mathrm{Zn}-\mathrm{O}$ bonds ${ }^{43}$.

Dynamic light scattering (DLS) is a relatively robust and economical technique to measure the average size and size distribution of green synthesized nanoparticles and nanocomposites. Mainly, DLS provides larger values because of the hydrodynamic shell, which is dependent on the structure, shape, and roughness of the particles ${ }^{44}$. According to Stokes-Einstein (SE) equation, the measured diffusion coefficients are related to the hydrodynamic radius as: $D=k_{B} T / 6 \pi \eta R_{h}$, where $k_{B}$ is Boltzmann's constant $\left(1.38 \times 10^{-23} \mathrm{~J} / \mathrm{K}\right), T$ is the temperature, $\eta$ is the viscosity of the suspension medium, and $R_{h}$ is the hydrodynamic radius ${ }^{45}$. There was an increase in the size of nanocomposites with the addition of silver to ZnO-GB (Figure 3a-d). The increase in the size can be caused by the formation of Ag NPs on the

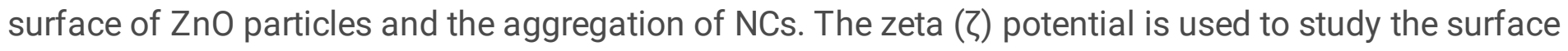
charges and stability of nano- or submicronic particles. The biomolecules from the GB extract were involved in reducing and stabilizing nanoparticles and nanocomposites. The zeta potential was calculated by dispersing the particles in water as the dispersion medium. The values of zeta potential were correlated with their stabilities: 0 to \pm 5 (rapid coagulation), \pm 10 to \pm 30 (incipient stability), \pm 30 to \pm 40 (moderate stability), \pm 40 to \pm 60 (good stability), and $> \pm 61$ (excellent stability) ${ }^{46,47}$. The average zeta potential of green synthesized ZnO-GB after calcination was $+2.72 \mathrm{mV}$ indicating positively charged

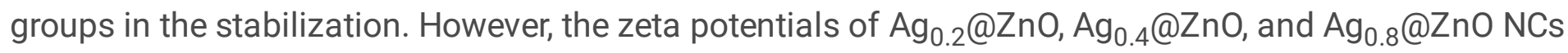
were $-16.4,-28.1$, and $-0.46 \mathrm{mV}$, respectively (Figure 3e-h). This shows that with the increase in the

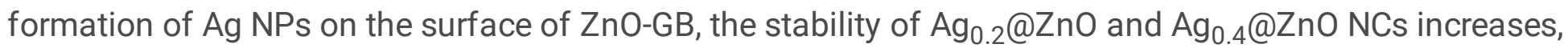
whereas the stability decreases with $\mathrm{Ag}_{0.8} @ Z n O N C$.

The surface morphology of ZnO-GB and various Ag@ZnO NCs, green synthesized by GB extract, were identified using FE-SEM. It can be noticed that most of the ZnO-GB showed clusters of ellipsoidal-like morphology on a submicronic scale. The ellipsoidal-like particles were in the size of $0.7 \pm 0.13$ and $0.38 \pm$ $0.075 \mu \mathrm{m}$ (length $\times$ width) (Figs. $4 \mathrm{a}$ and b). Remarkably, all ZnO-GB were almost identical in dimension, and the surface looks puffy with an irregular pattern of pillar ridges. There was a slight agglomeration of particles due to the slightly higher surface area and durable affinity among ZnO-GB particles ${ }^{46}$. Different 
morphologies of ZnO NPs, for example, nanospheres, nanoflower, nanoflakes, nanobelt, nanorods, nanowires, nanoneedles, nanotubes, and nanorings, can be synthesized by controlling the synthesis parameters ${ }^{20,48,49}$. The addition of silver with GB extract formed spherical Ag NPs i.e., $0.06 \pm 0.011,0.09$ \pm 0.04, $0.14 \pm 0.045 \mu \mathrm{m}$ for Ag $0.2 @ Z n O, \mathrm{Ag}_{0.4} @ Z n O$, and Ag $0.8 @ Z n O N C s$, respectively, on the surface of $\mathrm{ZnO}$ particles. There was no significant change in the morphology of Ag@ZnO NCs except with the size of embedded Ag NPs on the surface of $\mathrm{ZnO}$, which increased with the increase in the silver content added to the NCs (Figure 4c-h).

The elemental composition of ZnO-GB and Ag@ZnO NCs were analyzed using the FE-SEM-EDX spectra, as shown in Figure 5. The spectrum of ZnO-GB particles showed a low energy peak at approximately $0.533 \mathrm{keV}(\mathrm{O}-\mathrm{Ka})$ due to the presence of oxygen atom, and other peaks for zinc and carbon atoms appearing at about $1.02 \mathrm{keV}(\mathrm{Zn}-\mathrm{L} a), 8.6 \mathrm{keV}(\mathrm{Zn}-\mathrm{K} a), 9.5 \mathrm{keV}(\mathrm{Zn}-\mathrm{K} \beta)$, and $0.285 \mathrm{keV}(\mathrm{C}-\mathrm{K} a)$. In contrast, $\mathrm{Ag} @ Z n O N C s$ spectra contain intense low energy silver peaks at approximately $2.61 \mathrm{keV}(\mathrm{Ag}-\mathrm{Ka}), 3.0 \mathrm{keV}$ $(\mathrm{Ag}-\mathrm{L} a), 3.2 \mathrm{keV}(\mathrm{Ag}-\mathrm{L} \beta)$, and $3.4 \mathrm{keV}(\mathrm{Ag}-\mathrm{L} \beta 2)$ along with $\mathrm{Zn}, \mathrm{O}$, and $\mathrm{C}$ peaks ${ }^{50}$. The EDX quantified silver, and other elements content in various Ag@ZnO NCs is shown in Figure 5 (inset). The weight percent of silver increases from Ag ${ }_{0.2} @ Z n 0$ to Ag $0.8 @ Z n 0$, which infers the successful incorporation of silver as Ag NPs on $\mathrm{ZnO}-\mathrm{GB}^{51}$. Hence, the weight percentage of silver embedded on $\mathrm{ZnO}$ is proportional to the $\mathrm{Ag}$ concentration added to ZnO-GB in the preparation of different Ag@ZnO NCs.

FE-TEM analysis of ZnO-GB and different Ag@ZnO NCs are shown in Figure 6. Agglomeration of ellipsoidal-like ZnO-GB submicronic particles and the formation of spherical Ag NPs on the ZnO-GB surface was further verified by the FE-TEM results. It was found that ZnO-GB was about $0.6 \pm 0.11$ and $0.33 \pm 0.087 \mu \mathrm{m}$ (length $\times$ width), whereas $\mathrm{Ag}_{0.2} @ Z n 0, \mathrm{Ag}_{0.4} @ \mathrm{ZnO}$, and $\mathrm{Ag}_{0.8} @ \mathrm{ZnO} \mathrm{NCs}$ have Ag NPs in the size of $87 \pm 55,130 \pm 43$ and $160 \pm 55 \mathrm{~nm}$, respectively. Furthermore, these results corresponded to the poor correlation between the FE-TEM sizes and particle size distribution analysis. With the increase in the silver content in the NCs, there was an occurrence of large particles due to the aggregation of small or primary particles. The ' $d$ spacing of $\sim 0.281 \mathrm{~nm}$ between the adjacent lattice planes could be attributed to the (002) plane of ZnO-GB (Figure 6-d3). Similarly, the lattice fringes with $d=\sim 0.24 \mathrm{~nm}$ could be attributed to the (111) planes of Ag NPs (Figs. 6-b3 and c3). All these results confirmed the successful formation of Ag NPs on the surface of ZnO-GB. The $d$-spacing of (002) plane of ZnO-GB in Ag@ZnO NC is like that of undoped $\mathrm{ZnO}$, suggesting that $\mathrm{Ag}^{+}$ions are not incorporated into the $\mathrm{ZnO}$ lattice. SAED pattern of ZnO-GB clearly showed well-resolved diffraction rings indicating the polycrystallinity, and Ag NPs on ZnO-GB (Ag@ZnO NCs) showed bright spots indicating the monocrystalline nature.

Figure 7 shows the HAADF-STEM image of the $\mathrm{Ag}_{0.2} @ Z n O N C$ and its corresponding elemental composition (Zn-K, Zn-L, O-K, Ag-K, and Ag-L) by STEM-EDX mapping. These images confirm the successful embedment of Ag NPs on the surface of ZnO-GB.

XPS analysis was performed to clarify the chemical states of elements in Ag@ZnO NCs. The full scan survey of $\mathrm{Ag}_{0.2} @ \mathrm{ZnO} \mathrm{NC}$ shows the signals from $\mathrm{Zn}, \mathrm{O}$, and $\mathrm{Ag}$ elements with their corresponding atomic 
percent of $38.88,37.34$, and $1.63 \%$ in the range $0-1350 \mathrm{eV}$ (Figure 8a). Figure $8 \mathrm{~b}$ shows the highresolution spectra of Zn 2p. The peaks of Ag $_{0.2} @ Z n O ~ N C$ were located at 1021.28 eV and 1044.38 eV, which were ascribed to $Z n 2 p_{3 / 2}$ and $Z n 2 p_{1 / 2}$, respectively. These peaks confirm that the $Z n$ element exists in a divalent cation $\left(\mathrm{Zn}^{2+}\right)$ state in the NC. Figure $8 \mathrm{c}$ shows the high-resolution $01 \mathrm{~s}$ peak of Ag ${ }_{0.2} @ Z n O$ NC. The deconvoluted 0 1s peak shows two subpeaks at binding energies of 529.8 and 531.2 $\mathrm{eV}$ attributing to the lattice oxygen of ZnO-GB and dissociated oxygen or hydroxyl-like group on the surface of ZnO-GB, respectively 52,53 . The presence of surface hydroxyl groups acts as adsorption sites of dyes and reacts with photogenerated holes forming hydroxyl radicals by oxidation, which decomposes dyes during photodegradation ${ }^{54}$. Therefore, the presence of a surface hydroxyl group with $28.9 \%$ was one of the critical factors in the photodegradation process. Figure $8 \mathrm{~d}$ shows the high-resolution spectrum of Ag 3d photoelectron peaks of $\mathrm{Ag}_{0.2} @ Z n \mathrm{n}$ NC. The Ag 3d spectrum shows two peaks centered at 367.38 and $373.48 \mathrm{eV}$ ascribed to $\mathrm{Ag} 3 \mathrm{~d}_{5 / 2}$ and $\mathrm{Ag} 3 \mathrm{~d}_{3 / 2}$ transitions, respectively. The difference in the binding energy of $\sim 6.0 \mathrm{eV}$ between $\mathrm{Ag} 3 \mathrm{~d}_{5 / 2}$ and $\mathrm{Ag} 3 \mathrm{~d}_{3 / 2}$ peaks was the characteristic of metallic silver and consistent with the results of XRD analysis ${ }^{21,55}$.

Figure 9ashows the thermogravimetric (TG) analysis of ZnO-GB, Ag $0.2 @ \mathrm{ZnO}, \mathrm{Ag}_{0.4} @ \mathrm{ZnO}$, and $\mathrm{Ag}_{0.8} @ \mathrm{ZnO}$ NCs. TG analysis was performed from room temperature to $800^{\circ} \mathrm{C}$ at a rate of $20^{\circ} \mathrm{C} / \mathrm{min}$ in a nitrogen atmosphere to demonstrate the thermal stabilities of ZnO-GB and Ag@ZnO NCs. The overall weight loss for all samples was very minimal. A total weight loss of 3.0, 1.6, 0.3 , and $0.7 \%$ occurred for ZnO-GB, Ag $0.2 @ Z n O, A_{0.4} @ Z n O$, and Ag $_{0.8} @ Z n O N C s$, respectively, and all NCs are highly thermally stable. In ZnO$\mathrm{GB}$, at the low-temperature range (up to $90^{\circ} \mathrm{C}$ ), the weight seems to have increased due to the $\mathrm{OH}$ bonding with the reaction with moisture. Further, the weight loss up to $350^{\circ} \mathrm{C}$, accounting for $\sim 0.83 \%$ was attributed to the loss of $\mathrm{H}_{2} \mathrm{O}$ molecules and evaporation of gases that were physically and chemically adsorbed on the surface of ZnO-GB particles ${ }^{56}$. This explains that ZnO-GB absorbs nitrogen and slowly releases them over a period, which indicates that $\mathrm{ZnO}-\mathrm{GB}$ was pure and very porous in nature ${ }^{57}$. The weight loss accounting $\sim 2.1 \%$ from 350 to $700^{\circ} \mathrm{C}$ was higher with ZnO-GB, this could be due to the thermal decomposition of biomolecules of GB extracts, such as phenolic compounds and other metabolites. Above $700{ }^{\circ} \mathrm{C}$, there was no significant weight loss in ZnO-GB. A similar decomposition pattern was observed with $\mathrm{Ag}_{0.2} @ Z n O N C$, however, the embedment of silver slightly improved the thermal stability and decomposition of $\mathrm{Ag}_{0.2} @ \mathrm{ZnO} \mathrm{NC}$ compared to that of ZnO-GB.In $\mathrm{Ag}_{0.4} @ \mathrm{ZnO}$, and $\mathrm{Ag}_{0.8} @ Z \mathrm{ZnO} \mathrm{NCs}$, the continuous decrease in weight in the nitrogen environment was attributed probably to the oxygen out-diffusion from the $\mathrm{ZnO}$ matrix resulting in the formation of oxygen-deficient $\mathrm{ZnO}$ compound $\left(\mathrm{ZnO}_{1-\delta}\right)$.

$\mathrm{PL}$ spectrum is a valuable tool to investigate the state of photogenerated $\mathrm{e} \otimes / \mathrm{h}^{+}$pairs and the defects of metal/semiconductor nanocomposites ${ }^{58}$. Figure $9 \mathrm{~b}$ shows the PL spectra of ZnO-GB and Ag@ZnO NCs at room temperature. There are two distinct emission peaks at the UV region $(\sim 380 \mathrm{~nm})$ and visible region $(\sim 400-750 \mathrm{~nm})$. These emission peaks provide information about the recombination between charge carriers and defect levels ${ }^{17}$. The emission peak at $~ 380 \mathrm{~nm}$ in ZnO-GB corresponds to near band edge 
emission (NBE), attributed to bandgap excitation ${ }^{59}$. The broad band emission extending from $\sim 400 \mathrm{~nm}$ to $750 \mathrm{~nm}$ in the whole visible spectrum can be from deep-level emission (DLE), i.e., because of crystal defects like Zn-interstitials and oxygen vacancies ${ }^{43,60}$. All Ag@ZnO NCs showed decreased PL intensity than $\mathrm{ZnO}-\mathrm{GB}$, which suggests that the decrease in the recombination rate of photoinduced electrons and holes with the embedment of Ag NPs favoring the photocatalytic activity than ZnO-GB ${ }^{61}$. The PL intensity of $\mathrm{Ag}_{0.2} @ \mathrm{ZnO} \mathrm{NCs}$ decreased drastically with the increase in the silver content in the NCs providing the separation of photoinduced $\mathrm{e} / \mathrm{h} \mathrm{h}^{+}$pairs and inhibiting the recombination of photoinduced pairs ${ }^{62,63}$. However, at $\mathrm{Ag}_{0.8} @ \mathrm{ZnO} \mathrm{NC}$, with the increase in the Ag concentration, there was no increase in the PL intensity and overlapped with the peaks of $\mathrm{Ag}_{0.4} @ \mathrm{ZnO} \mathrm{NC}$, suggesting the formation of new recombination centers, which are unfavorable to the separation of photoinduced pairs ${ }^{58,64}$. Thus, $\mathrm{Ag}_{0.8} @ Z \mathrm{ZnO} \mathrm{NCs}$ exhibited the lowest PL intensity as that of $\mathrm{Ag}_{0.4} @ \mathrm{ZnO} \mathrm{NC}$ because excess addition of silver as Ag NPs in Ag, ${ }_{0.8} @ Z n O N C$ was unfavorable for charge separation.

To determine the structural and adsorption parameters of ZnO-GB and Ag $_{0.2} @ Z n O ~ N C$, nitrogen $\left(\mathrm{N}_{2}\right)$ adsorption-desorption isotherms at $77 \mathrm{~K}$ were recorded. Figure 10 a shows the $\mathrm{N}_{2}$ adsorption-desorption isotherms of ZnO-GB and Ag $_{0.2} @ Z n O N C$. According to IUPAC classification, these curves obtained for evaluating surface area were approximately identical to that of Type IV isotherm with $\mathrm{H}_{3}$ hysteresis loop ${ }^{65}$. The well-defined inflection around relative pressure $\left(P / P_{0}\right)$ of $0.5-0.9$ indicates the presence of a heterogeneously distributed mesoporous nature of particles ${ }^{66}$. The BET surface area $\left(S_{B E T}\right)$ was determined from isotherms using the BET equation ${ }^{67}$. The values of $S_{B E T}$ were found to be 11.77 and 7.5 $\mathrm{m}^{2} / \mathrm{g}$ for ZnO-GB and $\mathrm{Ag}_{0.2} @ \mathrm{ZnO} \mathrm{NC}$, respectively, and the mesoporous material contains narrow pores that hinder the movement of nitrogen and limits the adsorption. The $S_{B E T}$ of $\mathrm{Ag}_{0.2} @ Z n O N C$ decreased with the embedment of Ag NPs than ZnO-GB, revealing that the formed Ag NPs could have occupied and blocked the pores of $\mathrm{ZnO}-\mathrm{GB}$. Figure 10b shows the pore size distribution curve obtained using the Barrett-Joyner-Halenda (BJH) model. It could be seen that most of the pores were in the size range of 2-40 nm, which provides evidence for the mesoporosity framework of ZnO-GB and $\mathrm{Ag}_{0.2} @ Z n \mathrm{nO}$ N.The $\mathrm{BHJ}$ average pore sizes of ZnO-GB and $\mathrm{Ag}_{0.2} @ \mathrm{ZnO} \mathrm{NC}$ were 9.52 and $11.57 \mathrm{~nm}$, respectively, and the calculated mean pore volumes were 0.027 and $0.023 \mathrm{~cm}^{3} / \mathrm{g}$. The $S_{B E T}$, BHJ mean pore size, and porevolume of $\mathrm{Ag}_{0.2} @ \mathrm{ZnO} \mathrm{NC}$ were lower than that of $\mathrm{ZnO}-\mathrm{GB}$ because of the embedment of Ag NPs on the surface of ZnO-GB.

\section{Mechanism of photocatalytic activity}

The schematic diagram of the photocatalytic degradation of dyes MB and CR by ZnO-GB and Ag@ZnO $\mathrm{NCs}$ is proposed in Figure 10c. The advanced oxidation processes (AOPs) generate ROS of highly reactive

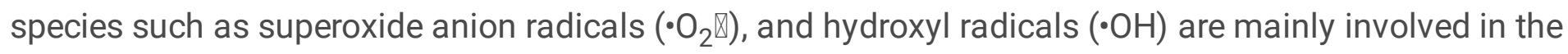
degradation and mineralization of dyes into carbon dioxide $\left(\mathrm{CO}_{2}\right)$ and water ${ }^{68}$. 
When $\mathrm{ZnO}$ is irradiated by the UV light of the simulated solar lamp, electrons in the valence band (VB) get excited to the conduction band (CB), leaving behind holes in the $\mathrm{VB}^{69}$. These photogenerated electrons get transferred to the Ag NPs as the CB energy level of ZnO-GB is higher than the Fermi level $\left(\mathrm{E}_{\mathrm{FM}}\right)$ of metallic Ag, which hinders the recombination and extends the lifetime of photogenerated $\left(\mathrm{e} / / \mathrm{h}^{+}\right)$pairs, whereas Ag NPs in the NCs absorb visible light undergo surface plasmon resonance (SPR), and these excited electrons in the 3d orbit of Ag NPs get easily transferred to CB of ZnO-GB owing to the interface effect of $\mathrm{Ag} / \mathrm{ZnO}$ heterojunctions, yielding more superoxide anion radicals. The holes formed by the excitation of electrons will generate $\cdot \mathrm{OH}$ radicals by oxidation of hydroxyl ions. Thus, the as-formed superoxide anion radicals and hydroxyl radicals are mainly responsible for the effective mineralization of dyes into $\mathrm{CO}_{2}$ and water ${ }^{70,71}$. The increase of silver amount on the surface of $\mathrm{ZnO}-\mathrm{GB}$ decreases the photocatalytic degradation efficiency. The decrease in the photocatalytic degradation by $\mathrm{Ag}_{0.4} @ \mathrm{ZnO}$ and $\mathrm{Ag}_{0.8} @ \mathrm{ZnO} \mathrm{NCs}$ could be due to the hindrance in the absorption of light by the excess of Ag NPs, which is in agreement with the PL results.

\section{Applications of ZnO-GB and Ag@ZnO NCs}

\section{Photocatalytic degradation of dyes}

Photocatalysis happens on the surface of the photocatalyst, and the photocatalytic performance of ZnOGB was ameliorated by increasing surface-to-volume ratio and by modifying the band structure by the incorporation of Ag NPs to improve the visible-light absorption properties and thereby efficiently restricting the recombination of photogenerated $\left(\mathrm{e} \mathbb{\mathrm { A }} / \mathrm{h}^{+}\right)$pairs ${ }^{17,72,73}$. The photocatalytic properties of ZnO-GB and Ag@ZnO NCs were evaluated via the degradation of dyes MB (cationic) and CR (anionic) under the simulated solar lamp. Figure 11a shows the UV-vis absorption spectra of the degradation of MB with time in the presence of ZnO-GB and Ag@ZnO NCs. Figure 11c shows the photocatalytic degradation $\left(C_{t} / C_{0}\right)$ as a function of time, where $C_{t}$ is the concentration of $M B$ at time " $t$ ", and $C_{0}$ is the initial concentration. The experimental solution containing the MB $(1.0 \mathrm{mg} / 100 \mathrm{ml})$ and photocatalyst $(0.1 \% \mathrm{w} / \mathrm{v})$ was allowed for the adsorption-desorption equilibrium in the dark for $30 \mathrm{~min}$, and the $\mathrm{MB}$ dye in the range of $3.0 \pm 2.5-12.7 \pm 4.2 \%$ was adsorbed on ZnO-GB and Ag@ZnO NCs. The increase of silver content as Ag NPs on $\mathrm{ZnO}$ increased the adsorption of MB dye on its surface. Moreover, the strong MB dye adsorption capacities by Ag@NCs in the dark improved their photocatalytic performances in terms of their decolorization and degradation processes ${ }^{74}$. The degradation percentage of MB by ZnO-GB, Ag $0.2 @ Z n 0, A_{0.4} @ Z n 0$, and Ag ${ }_{0.8} @ Z n O N C s$ were $89.4 \pm 2.2,99.2 \pm 0.34,97.6 \pm 0.91$, and $96.0 \pm 0.1 .5 \%$, respectively after irradiation for $90 \mathrm{~min}$ (Figure 11e). Ag $0.2 @ \mathrm{ZnO}$ as photocatalysts showed higher photocatalytic degradative activity than other NCs and ZnO-GB, and it showed $100 \%$ degradation in 90 min. However, other Ag@ZnO NCs and ZnO-GB showed 100\% photocatalytic degradation in 120 min. The higher photocatalytic activity by $\mathrm{Ag}_{0.2} @ \mathrm{ZnO} \mathrm{NC}$ suggests that adding silver to ZnO-GB improves the photocatalytic activity significantly. 
Similarly, the UV-vis absorption spectra of the degradation of CR dye with time by ZnO-GB and different Ag@ZnO NC were shown in Figure 11b. The $C_{t} / C_{0}$ degradation of $C R$ versus time was shown in Figure $11 \mathrm{~d}$. After incubation at dark for attaining adsorption-desorption equilibrium, CR dye of $26.0 \pm 0.77-$ $74.4 \pm 1.3 \%$ was adsorbed onto ZnO-GB and Ag@ZnO NCs. ZnO-GB had a strong adsorption ability of CR on its surface, however, with an increase in the silver amount, the adsorption of CR on the surface of NCs decreases. Moreover, the strong adsorption capacities of ZnO-GB and Ag@ZnO NCs in the dark improved their photocatalytic performances in their decolorization and degradation processes. The degradation

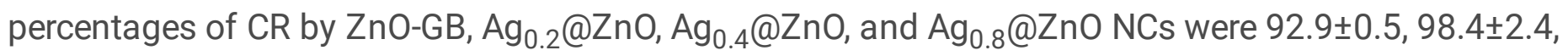
$92.5 \pm 1.5$, and $86.1 \pm 1.5 \%$, respectively after irradiation for $55 \mathrm{~min}$ (Figure 11f) (Table 1). Analogous to MB degradation, the degradation of $\mathrm{CR}$ by $\mathrm{Ag}_{0.2} @ \mathrm{ZnO} \mathrm{NC}$ was higher than other Ag@ZnO NCs and ZnO-GB. The photolysis of dyes without photocatalyst was also determined. Both dyes are barely degraded without photocatalyst, which indicates that both MB and CR dyes are stable in the aqueous environment under simulated solar irradiation. However, $\mathrm{CR}$ appears to be more stable than MB under the experimental conditions. There was photolysis of $26.97 \%$ and $4.26 \%$ for MB and CR dyes, respectively, after irradiation for 150 and 210 min (Supplementary Figure 1 and 2).

\section{Antibacterial assay}

Figure 12 shows the antibacterial activity of ZnO-GB and other Ag@ZnO NCs against Gram-positive (S. aureus) and Gram-negative (E. coli) bacteria. The antibacterial activity was evaluated using the agar well diffusion method, which shows that both E. coli and S. aureus were susceptible to all Ag@ZnO NCs. However, E. coli showed slight resistance towards the antimicrobial activity by ZnO-GB and Ag@ZnO NCs. The zones of inhibitions (ZOIs) for Ag $0.2 @ Z n O, A_{0.4} @ Z n O$, and Ag ${ }_{0.8} @ Z n O N C s$ were 11.0 \pm 0.4, $11.4 \pm$ 0.5 , and $11.3 \pm 0.6 \mathrm{~mm}$, respectively, for $E$. coli, whereas it was $13.8 \pm 0.6,14.4 \pm 1.0$, and $14.6 \pm 0.9 \mathrm{~mm}$ for $S$. aureus. Moreover, ZnO-GB showed a marginal ZOI of $10.2 \pm 0.4 \mathrm{~mm}$ only in S. aureus. The ZOls for the positive control (ampicillin) were $12.6 \pm 0.5$ and $25.6 \pm 0.7 \mathrm{~mm}$ for $E$. coli and $S$. aureus, respectively. Our previous study found that GB extract does not possess antibacterial activity against $E$. coli and $S$. aureus $^{21}$. Thus, all Ag@ZnO NCs at a $2 \mathrm{mg}(0.05 \mathrm{~mL})$ concentration exhibited broad-spectrum antibacterial activity against both $E$. coli and $S$. aureus. The antibacterial activity of the ZnO-GB is most likely due to the release of ROS on the surface of $\mathrm{ZnO}-\mathrm{GB}$, which causes the disruption of the bacterial membrane, in particular, the production of hydrogen peroxide $\left(\mathrm{H}_{2} \mathrm{O}_{2}\right)$ penetrates the cell membrane and kills the microorganism ${ }^{75,76}$. Gunalan et al. (2012) demonstrated the antibacterial activity of Aloe leaf extract-mediated synthesis of nano-ZnO against $S$. aureus, Serratia marcescens, Proteus mirabilis, and Citrobacter freundii ${ }^{77}$. Even Ag NPs in Ag@ZnO NCs can cause membrane permeation and bacterial ROS production for the synergistic antibacterial activity with ZnO-GB particles in the nanocomposite ${ }^{78,79}$. Zare, et al. ${ }^{80}$ evaluated the antibacterial potency of ZnO-Ag NC on bacteria. They proposed that physical interaction with bacterial cells causes disruption of cell membrane and oxidization of cell components for exhibiting broad-spectrum antibacterial activity against multidrug resistant bacteria. 


\section{Conclusions}

The green synthesis of zinc oxide particles (ZnO-GB) using goji berry extract and subsequent calcination in air produced mesoporous elliptical-like morphology with $0.59 \mu \mathrm{m}$ (length), and $0.33 \mu \mathrm{m}$ (width) was found to be of hexagonal wurtzite crystal structure. The formation of silver nanoparticles on the surface of ZnO-GB in the formation of Ag@ZnO nanocomposites using the GB extract provides a green method of synthesizing highly porous metal/semiconductor NCs. The presence of polyphenols in the GB extract acts as both reducing and capping/stabilizing agents in preparing nanoparticles and/or nanocomposites. The as-prepared Ag@ZnO NCs were characterized by several techniques, such as FT-IR, XRD, FE-SEM, TEM, EDS, XPS, and UV-vis spectroscopy. The XRD analysis and SEM-EDX and TEM micrographs confirmed the formation of Ag NPs on the surface of ZnO. The photocatalytic activity of $\mathrm{Ag}_{0.2} @ \mathrm{ZnO}$ nanocomposite towards both $\mathrm{MB}$ and $\mathrm{CR}$ degradation in an aqueous medium was found to be higher than that of ZnO-GB and other Ag@ZnO NCs at room temperature. The presence of Ag on the surface of $\mathrm{ZnO}$ promotes the separation of photogenerated charge carriers and enhances photocatalysis. In addition, they also showed good antibacterial activity against Staphylococcus aureus and Escherichia coli. The photocatalysis and antibacterial activity of $\mathrm{Ag}_{0.2} @ \mathrm{ZnO}$ are remarkably improved due to the generation of abundant ROS than that of ZnO-GB and other Ag@ZnO NCs. This simple green synthetic methodology provides unique morphological metal/semiconductor nanocomposites for wastewater treatment by photocatalysis and antimicrobial therapeutics.

\section{Declarations}

\section{Acknowledgments}

This work was supported by the King Abdullah Scholarship Program (2018), Ministry of Education, Saudi Arabia, and the Basic Science Research Program through the National Research Foundation of Korea (NRF) (\#2019R1I1A3A01062440, \#2020R1A2C1012586, \& \#2020R1A6A1A03044512) funded by the Korean Ministry of Education, Science and Technology. The authors thank the Core Research Support Center for Natural Products and Medical Materials (CRCNM) for instrumentation support.

\section{Author Contributions}

A. A. S: Conceptualization, Methodology, Validation, Formal analysis, Writing - Original draft \& Editing. K. B. N: Conceptualization, methodology, Validation, Data analysis, Writing, Work administration, Writing Final draft, review \& Editing, \& Supervision. M. E. K: Data analysis, Writing - Review \& Editing. S. S. H: Resources, Validation, Final draft - review \& Editing \& Supervision.

\section{Competing Interests}

The authors declare competing interests.

\section{Data availability statement}


Data available on request from the authors

\section{References}

1. Alrousan, D., Afkhami, A., Bani-Melhem, K. \& Dunlop, P. Organic Degradation Potential of Real Greywater Using TiO2-Based Advanced Oxidation Processes. Water 12, 2811 (2020).

2. Esplugas, S., Giménez, J., Contreras, S., Pascual, E. \& Rodríguez, M. Comparison of different advanced oxidation processes for phenol degradation. Water Research 36, 1034-1042, doi:https://doi.org/10.1016/S0043-1354(01)00301-3 (2002).

3. Ameta, N. S., Jyoti; Sharma, Sanyogita; Kumar, Sudhish; Punjabi, B Pinki. Copper modified iron oxide as heterogeneous photo-Fenton reagent for the degradation of coomasie brilliant blue R-250. Indian Journal of Chemistry 51A, 943-948 (2012).

4. Ramesha, G. K., Vijaya Kumara, A., Muralidhara, H. B. \& Sampath, S. Graphene and graphene oxide as effective adsorbents toward anionic and cationic dyes. Journal of Colloid and Interface Science 361, 270-277, doi:https://doi.org/10.1016/j.jcis.2011.05.050 (2011).

5. Chiu, Y.-H., Chang, T.-F. M., Chen, C.-Y., Sone, M. \& Hsu, Y.-J. Mechanistic Insights into Photodegradation of Organic Dyes Using Heterostructure Photocatalysts. Catalysts $\mathbf{9}$, doi:10.3390/catal9050430 (2019).

6. Rafiq, A. et al. Photocatalytic degradation of dyes using semiconductor photocatalysts to clean industrial water pollution. Journal of Industrial and Engineering Chemistry 97, 111-128, doi:https://doi.org/10.1016/j.jiec.2021.02.017 (2021).

7. Umar, M. \& Aziz, H. A. Photocatalytic degradation of organic pollutants in water. Organic pollutantsmonitoring, risk and treatment 8, 196-197 (2013).

8. Aramendía, M. A., Marinas, A., Marinas, J. M., Moreno, J. M. \& Urbano, F. J. Photocatalytic degradation of herbicide fluroxypyr in aqueous suspension of $\mathrm{TiO}_{2}$. Catalysis Today 101, 187-193, doi:https://doi.org/10.1016/j.cattod.2005.03.063 (2005).

9. Lee, Y. Y. et al. Visible-Light Driven Photocatalytic Degradation of Organic Dyes over Ordered Mesoporous CdxZn1-xS Materials. The Journal of Physical Chemistry C 121, 5137-5144, doi:10.1021/acs.jpcc.7b00038 (2017).

10. Fagier, M. A. Plant-Mediated Biosynthesis and Photocatalysis Activities of Zinc Oxide Nanoparticles: A Prospect towards Dyes Mineralization. Journal of Nanotechnology 2021 (2021).

11. Gupta, J., Bhargava, P. \& Bahadur, D. Morphology dependent photocatalytic and magnetic properties of ZnO nanostructures. Physica B: Condensed Matter 448, 16-19 (2014).

12. Schubert, E. F. Doping in III-V semiconductors. (E. Fred Schubert, 2015).

13. Bhardwaj, D. \& Singh, R. Green biomimetic synthesis of Ag-TiO 2 nanocomposite using Origanum majorana leaf extract under sonication and their biological activities. Bioresources and Bioprocessing 8, 1-12 (2021). 
14. Demissie, M. G., Sabir, F. K., Edossa, G. D. \& Gonfa, B. A. Synthesis of zinc oxide nanoparticles using leaf extract of lippia adoensis (koseret) and evaluation of its antibacterial activity. Journal of Chemistry 2020 (2020).

15. Sadiq, H. et al. Green synthesis of $\mathrm{ZnO}$ nanoparticles from Syzygium Cumini leaves extract with robust photocatalysis applications. Journal of Molecular Liquids 335, 116567 (2021).

16. He, W. et al. Photogenerated Charge Carriers and Reactive Oxygen Species in ZnO/Au Hybrid Nanostructures with Enhanced Photocatalytic and Antibacterial Activity. Journal of the American Chemical Society 136, 750-757, doi:10.1021/ja410800y (2014).

17. Gupta, J., Mohapatra, J. \& Bahadur, D. Visible light driven mesoporous Ag-embedded ZnO nanocomposites: reactive oxygen species enhanced photocatalysis, bacterial inhibition and photodynamic therapy. Dalton Transactions 46, 685-696, doi:10.1039/C6DT03713E (2017).

18. Singh, J. et al. 'Green'synthesis of metals and their oxide nanoparticles: applications for environmental remediation. Journal of nanobiotechnology 16, 1-24 (2018).

19. Dong, C. et al. Wolfberry fruit (Lycium barbarum) extract mediated novel route for the green synthesis of silver nanoparticles. Optik 130, 162-170, doi:https://doi.org/10.1016/j.jileo.2016.11.010 (2017).

20. Chauhan, A. et al. Photocatalytic dye degradation and antimicrobial activities of Pure and Ag-doped ZnO using Cannabis sativa leaf extract. Scientific Reports 10, 7881, doi:10.1038/s41598-020-644190 (2020).

21. Ahmed Sharwani, A., Badri Narayanan, K., Ehtisham Khan, M. \& Soo Han, S. Sustainable fabrication of silver-titania nanocomposites using goji berry (Lycium barbarum L.) fruit extract and their photocatalytic and antibacterial applications. Arabian Journal of Chemistry 14, 103456, doi:https://doi.org/10.1016/j.arabjc.2021.103456 (2021).

22. Narayanan, K. B., Kim, H. D. \& Han, S. S. Biocompatibility and hemocompatibility of hydrothermally derived reduced graphene oxide using soluble starch as a reducing agent. Colloids and Surfaces B: Biointerfaces 185, 110579, doi:https://doi.org/10.1016/j.colsurfb.2019.110579 (2020).

23. Narayanan, K. B., Park, G. T. \& Han, S. S. Antibacterial properties of starch-reduced graphene oxidepolyiodide nanocomposite. Food Chemistry 342, 128385, doi:https://doi.org/10.1016/j.foodchem.2020.128385 (2021).

24. Çolak, H. \& Karaköse, E. Green synthesis and characterization of nanostructured ZnO thin films using Citrus aurantifolia (lemon) peel extract by spin-coating method. Journal of Alloys and Compounds 690, 658-662, doi:https://doi.org/10.1016/j.jallcom.2016.08.090 (2017).

25. Gao, Y., Xu, D., Ren, D., Zeng, K. \& Wu, X. Green synthesis of zinc oxide nanoparticles using Citrus sinensis peel extract and application to strawberry preservation: A comparison study. LWT 126, 109297, doi:https://doi.org/10.1016/j.lwt.2020.109297 (2020).

26. Narayanan, K. B. \& Sakthivel, N. Green synthesis of biogenic metal nanoparticles by terrestrial and aquatic phototrophic and heterotrophic eukaryotes and biocompatible agents. Advances in Colloid and Interface Science 169, 59-79, doi:https://doi.org/10.1016/j.cis.2011.08.004 (2011). 
27. González, A. L., Noguez, C., Beránek, J. \& Barnard, A. S. Size, Shape, Stability, and Color of Plasmonic Silver Nanoparticles. The Journal of Physical Chemistry C 118, 9128-9136, doi:10.1021/jp5018168 (2014).

28. Ahmad, N. et al. Rapid synthesis of silver nanoparticles using dried medicinal plant of basil. Colloids and Surfaces B: Biointerfaces 81, 81-86, doi:https://doi.org/10.1016/j.colsurfb.2010.06.029 (2010).

29. Makarov, V. V. et al. "Green” Nanotechnologies: Synthesis of Metal Nanoparticles Using Plants. ActaNaturae 6, 35-44, doi:10.32607/20758251-2014-6-1-35-44 (2014).

30. Rai, M. et al. Biogenic Silver Nanoparticles: What We Know and What Do We Need to Know? Nanomaterials 11, 2901 (2021).

31. El-Seedi, H. R. et al. Metal nanoparticles fabricated by green chemistry using natural extracts: biosynthesis, mechanisms, and applications. RSC advances 9, 24539-24559 (2019).

32. Choudhary, M. K., Kataria, J., Bhardwaj, V. K. \& Sharma, S. Green biomimetic preparation of efficient $\mathrm{Ag}-\mathrm{ZnO}$ heterojunctions with excellent photocatalytic performance under solar light irradiation: a novel biogenic-deposition-precipitation approach. Nanoscale Advances 1, 1035-1044, doi:10.1039/C8NA00318A (2019).

33. Saboor, A., Shah, S. M. \& Hussain, H. Band gap tuning and applications of $\mathrm{ZnO}$ nanorods in hybrid solar cell: Ag-doped verses Nd-doped ZnO nanorods. Materials Science in Semiconductor Processing 93, 215-225, doi:https://doi.org/10.1016/j.mssp.2019.01.009 (2019).

34. Koch, U., Fojtik, A., Weller, H. \& Henglein, A. Photochemistry of semiconductor colloids. Preparation of extremely small $\mathrm{ZnO}$ particles, fluorescence phenomena and size quantization effects. Chemical Physics Letters 122, 507-510, doi:https://doi.org/10.1016/0009-2614(85)87255-9 (1985).

35. Soylu, M. \& Savas, O. Electrical and optical properties of $\mathrm{ZnO} / \mathrm{Si}$ heterojunctions as a function of the Mg dopant content. Materials Science in Semiconductor Processing 29, 76-82 (2015).

36. Qu, J., Luo, C. \& Hou, J. Synthesis of ZnO nanoparticles from Zn-hyperaccumulator (Sedum alfredii Hance) plants. Micro \& Nano Letters 6, 174-176 (2011).

37. Qu, J., Yuan, X., Wang, X. \& Shao, P. Zinc accumulation and synthesis of ZnO nanoparticles using Physalis alkekengi L. Environmental pollution 159, 1783-1788 (2011).

38. Zaid, M. H. M., Matori, K. A., Yaakob, Y. \& Alibe, I. M. Simple thermal treatment approach for the synthesis of a-Zn2SiO4 nanoparticles. Optics \& Laser Technology 140, 106991, doi:https://doi.org/10.1016/j.optlastec.2021.106991 (2021).

39. Panchal, P. et al. Biogenic mediated Ag/ZnO nanocomposites for photocatalytic and antibacterial activities towards disinfection of water. Journal of Colloid and Interface Science 563, 370-380, doi:https://doi.org/10.1016/j.jcis.2019.12.079 (2020).

40. Mathlouthi, M. \& Koenig, J. L. in Advances in Carbohydrate Chemistry and Biochemistry Vol. 44 (eds R. Stuart Tipson \& Derek Horton) 7-89 (Academic Press, 1987).

41. Santos, D. I. et al. Fourier Transform Infrared (FT-IR) Spectroscopy as a Possible Rapid Tool to Evaluate Abiotic Stress Effects on Pineapple By-Products. Applied Sciences 9, doi:10.3390/app9194141 (2019). 
42. Dabbebi, R., Baklouti, S., de Aguiar, J. L. B., Pacheco-Torgal, F. \& Samet, B. Investigations of geopolymeric mixtures based on phosphate washing waste. Science and Technology of Materials 30,1-5\%@2603-6363 (2018).

43. Sajjad, M. et al. Structural and optical properties of pure and copper doped zinc oxide nanoparticles. Results in Physics 9, 1301-1309, doi:https://doi.org/10.1016/j.rinp.2018.04.010 (2018).

44. Kätzel, U. et al. Dynamic Light Scattering for the Characterization of Polydisperse Fractal Systems: I. Simulation of the Diffusional Behavior. Particle \& Particle Systems Characterization 25, 9-18, doi:https://doi.org/10.1002/ppsc.200700004 (2008).

45. Zmpitas, J. \& Gross, J. Modified Stokes-Einstein Equation for Molecular Self-Diffusion Based on Entropy Scaling. Industrial \& Engineering Chemistry Research 60, 4453-4459, doi:10.1021/acs.iecr.0c06090 (2021).

46. Barzinjy, A. A. \& Azeez, H. H. Green synthesis and characterization of zinc oxide nanoparticles using Eucalyptus globulus Labill. leaf extract and zinc nitrate hexahydrate salt. SN Applied Sciences 2, 991, doi:10.1007/s42452-020-2813-1 (2020).

47. Kumar, A. \& Dixit, C. K. in Advances in Nanomedicine for the Delivery of Therapeutic Nucleic Acids (eds Surendra Nimesh, Ramesh Chandra, \& Nidhi Gupta) 43-58 (Woodhead Publishing, 2017).

48. Iravani, S. Green synthesis of metal nanoparticles using plants. Green Chemistry 13, 2638-2650, doi:10.1039/C1GC15386B (2011).

49. Jayappa, M. D. et al. Green synthesis of zinc oxide nanoparticles from the leaf, stem and in vitro grown callus of Mussaenda frondosa L.: characterization and their applications. Applied Nanoscience 10, 3057-3074, doi:10.1007/s13204-020-01382-2 (2020).

50. Majeed Khan, M. A., Kumar, S., Ahamed, M., Alrokayan, S. A. \& AlSalhi, M. S. Structural and thermal studies of silver nanoparticles and electrical transport study of their thin films. Nanoscale Research Letters 6, 434, doi:10.1186/1556-276X-6-434 (2011).

51. Gurgur, E., Oluyamo, S. S., Adetuyi, A. O., Omotunde, O. I. \& Okoronkwo, A. E. Green synthesis of zinc oxide nanoparticles and zinc oxide-silver, zinc oxide-copper nanocomposites using Bridelia ferruginea as biotemplate. SN Applied Sciences 2, 911, doi:10.1007/s42452-020-2269-3 (2020).

52. Demirci, S. et al. Synthesis and characterization of $\mathrm{Ag}$ doped $\mathrm{TiO}_{2}$ heterojunction films and their photocatalytic performances. Applied Surface Science 390, 591-601, doi:https://doi.org/10.1016/j.apsusc.2016.08.145 (2016).

53. Xin, B., Jing, L., Ren, Z., Wang, B. \& Fu, H. Effects of simultaneously doped and deposited Ag on the photocatalytic activity and surface states of $\mathrm{TiO}_{2}$. The Journal of Physical Chemistry B 109, 28052809 (2005).

54. Avilés-García, O. et al. W and Mo doped TiO2: synthesis, characterization and photocatalytic activity. Fuel 198, 31-41 (2017).

55. Xiang, Q., Yu, J., Cheng, B. \& Ong, H. C. Microwave-Hydrothermal Preparation and Visible-Light Photoactivity of Plasmonic Photocatalyst $\mathrm{Ag}-\mathrm{TiO}_{2}$ Nanocomposite Hollow Spheres. Chemistry - An 
Asian Journal 5, 1466-1474, doi:https://doi.org/10.1002/asia.200900695 (2010).

56. Lee, C. R. et al. Synthesis and Ag recovery of nanosized ZnO powder by solution combustion process for photocatalytic applications. 9, 281-286 (2001).

57. Prashanth, G. K. et al. Comparison of anticancer activity of biocompatible ZnO nanoparticles prepared by solution combustion synthesis using aqueous leaf extracts of Abutilon indicum, Melia azedarach and Indigofera tinctoria as biofuels. Artificial Cells, Nanomedicine, and Biotechnology 46, 968-979, doi:10.1080/21691401.2017.1351982 (2018).

58. Lang, J. et al. Chemical precipitation synthesis and significant enhancement in photocatalytic activity of Ce-doped ZnO nanoparticles. Ceramics International 42, 14175-14181, doi:https://doi.org/10.1016/j.ceramint.2016.06.042 (2016).

59. Zhang, L. et al. On the structure and ultraviolet emission of terbium doped zinc oxide thin films on silicon after high temperature treatment. Results in Physics 32, 105121, doi:https://doi.org/10.1016/j.rinp.2021.105121 (2022).

60. Willander, M. et al. Luminescence from zinc oxide nanostructures and polymers and their hybrid devices. Materials 3, 2643-2667 (2010).

61. Kuo, S.-T., Tuan, W.-H., Shieh, J. \& Wang, S.-F. Effect of Ag on the Microstructure and Electrical Properties of ZnO. Journal of the European Ceramic Society 27, 4521-4527, doi:10.1016/j.jeurceramsoc.2007.02.215 (2007).

62. Vanheusden, K. et al. Mechanisms behind green photoluminescence in $\mathrm{ZnO}$ phosphor powders. Journal of Applied Physics 79, 7983-7990, doi:10.1063/1.362349 (1996).

63. Zheng, Y. et al. Photocatalytic Activity of Ag/ZnO Heterostructure Nanocatalyst: Correlation between Structure and Property. The Journal of Physical Chemistry C 112, 10773-10777, doi:10.1021/jp8027275 (2008).

64. Zhu, X. et al. Fabrication, characterization, and photocatalytic activity of anatase/rutile/ $\mathrm{SnO}_{2}$ nanocomposites. Journal of Materials Science: Materials in Electronics 30, 21210-21218, doi:10.1007/s10854-019-02494-4 (2019).

65. Sing, K. S. Reporting physisorption data for gas/solid systems with special reference to the determination of surface area and porosity (Recommendations 1984). Pure and applied chemistry 57, 603-619 (1985).

66. Devi, M. R. \& Kannan, C. Synthesis of unusual large pore Mg substituted alumino phosphate (MgAlP04) mesoporous molecular sieve and its catalytic activity. Journal of Applicable Chemistry 2 , 398-404 (2013).

67. Sing, K. S. Adsorption methods for the characterization of porous materials. Advances in colloid and interface science 76, 3-11 (1998).

68. Khataee, A. R. \& Kasiri, M. B. Photocatalytic degradation of organic dyes in the presence of nanostructured titanium dioxide: Influence of the chemical structure of dyes. Journal of Molecular Catalysis A: Chemical 328, 8-26, doi:https://doi.org/10.1016/j.molcata.2010.05.023 (2010). 
69. Pimpliskar, P. V., Motekar, S. C., Umarji, G. G., Lee, W. \& Arbuj, S. S. Synthesis of silver-loaded ZnO nanorods and their enhanced photocatalytic activity and photoconductivity study. Photochemical \& Photobiological Sciences 18, 1503-1511, doi:10.1039/C9PP00099B (2019).

70. Dhatshanamurthi, P. et al. Superficial sonication synthesis of a highly sunshine active metal modified $\mathrm{ZnO}$ photocatalyst and its multiple applications. Inorganic and Nano-Metal Chemistry, 112, doi:10.1080/24701556.2021.1980023 (2021).

71. Majumder, S., Basnet, P., Mukherjee, J. \& Chatterjee, S. Bio-capped facile synthesis of silver zinc oxide for photocatalytic degradation of Rhodamine $6 \mathrm{G}$ under visible-light irradiation. AIP Conference Proceedings 2265, 030093, doi:10.1063/5.0017417 (2020).

72. Alharthi, F. A. et al. Facile one-pot green synthesis of $\mathrm{Ag}-\mathrm{ZnO}$ Nanocomposites using potato peeland their Ag concentration dependent photocatalytic properties. Scientific Reports 10, 20229, doi:10.1038/s41598-020-77426-y (2020).

73. Essawy, A. A. Silver imprinted zinc oxide nanoparticles: Green synthetic approach, characterization and efficient sunlight-induced photocatalytic water detoxification. Journal of Cleaner Production 183, 1011-1020, doi:https://doi.org/10.1016/j.jclepro.2018.02.214 (2018).

74. Kumar, A., Sharma, G., Naushad, M., Singh, P. \& Kalia, S. Polyacrylamide/Ni0.02Zn0.980 Nanocomposite with High Solar Light Photocatalytic Activity and Efficient Adsorption Capacity for Toxic Dye Removal. Industrial \& Engineering Chemistry Research 53, 15549-15560, doi:10.1021/ie5018173 (2014).

75. Janaki, A. C., Sailatha, E. \& Gunasekaran, S. Synthesis, characteristics and antimicrobial activity of ZnO nanoparticles. Spectrochimica Acta Part A: Molecular and Biomolecular Spectroscopy 144, 1722 (2015).

76. Sarwar, S. et al. The antimicrobial activity of $\mathrm{ZnO}$ nanoparticles against Vibrio cholerae: Variation in response depends on biotype. Nanomedicine: Nanotechnology, Biology and Medicine 12, 1499-1509 (2016).

77. Gunalan, S., Sivaraj, R. \& Rajendran, V. Green synthesized ZnO nanoparticles against bacterial and fungal pathogens. Progress in Natural Science: Materials International 22, 693-700 (2012).

78. Domínguez, A. V., Algaba, R. A., Canturri, A. M., Villodres, Á. R. \& Smani, Y. Antibacterial activity of colloidal silver against gram-negative and gram-positive bacteria. Antibiotics 9 (2020).

79. Noohpisheh, Z., Amiri, H., Farhadi, S. \& Mohammadi-gholami, A. Green synthesis of Ag-ZnO nanocomposites using Trigonella foenum-graecum leaf extract and their antibacterial, antifungal, antioxidant and photocatalytic properties. Spectrochimica Acta Part A: Molecular and Biomolecular Spectroscopy 240, 118595, doi:https://doi.org/10.1016/j.saa.2020.118595 (2020).

80. Zare, M. et al. Novel Green Biomimetic Approach for Synthesis of ZnO-Ag Nanocomposite; Antimicrobial Activity against Food-borne Pathogen, Biocompatibility and Solar Photocatalysis. Scientific Reports 9, 8303, doi:10.1038/s41598-019-44309-w (2019).

\section{Tables}


Table 1. Green synthesis of Ag-ZnO NCs and their photocatalytic degradation of dyes and antibacterial activities

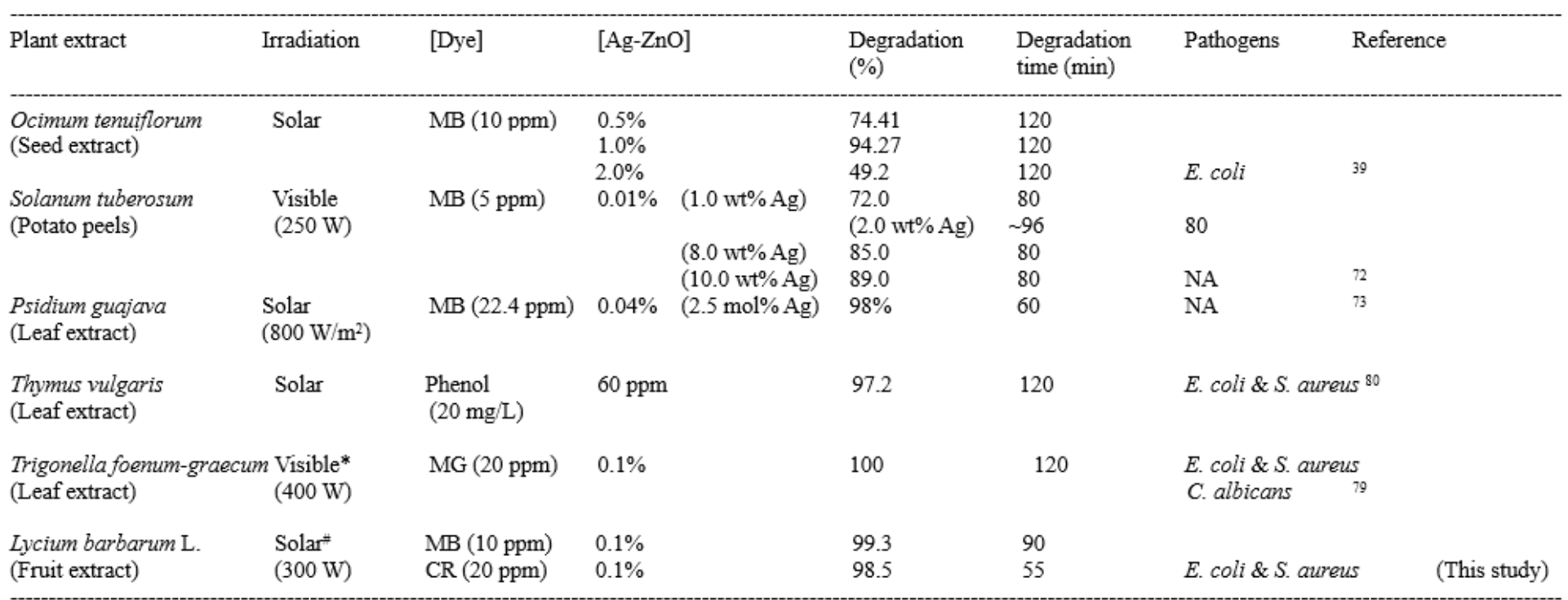

NA: Not applicable, MB: Methylente blue, MG: Malachite green, ${ }^{*} \mathrm{Hg}$ lamp $(\lambda>420 \mathrm{~nm}),{ }^{*}$ simulated solar lamp

\section{Figures}

\section{Figure 1}

Schematic diagram of the green synthesis of ZnO-GB and Ag@ZnO NCs.

\section{Figure 2}

(a) UV-vis DRS absorption spectra, (b) Tauc plots, (c) Powder XRD analyses and (d) FTIR spectra of ZnO$\mathrm{GB}, \mathrm{Ag}_{0.2} @ Z n \mathrm{Zn}, \mathrm{Ag}_{0.4} @ \mathrm{ZnO}$, and Ag $\mathrm{Ag}_{0.8} @ Z n O \mathrm{NCs}$.

\section{Figure 3}

(a-d) Dynamic light scattering of particle size distribution and (e-h) zeta potentials of ZnO-GB, Ag $g_{0.2} @ Z n 0$, Ago.4@Zno, and Ag $0.8 @ Z n O N C s$. 


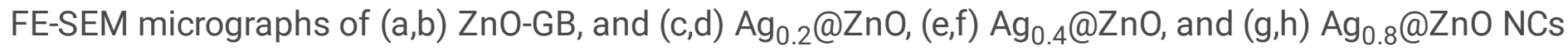
(Scale bar: $5 \mu \mathrm{m}$ (left) and $1 \mu \mathrm{m}$ (right)).

\section{Figure 5}

FE-SEM-EDX spectra of (a) ZnO-GB, (b) Ag $0.2 @ Z n O,(c)$ Ag $_{0.4} @ Z n O$, and (d) Ag $0.8 @ Z n O ~ N C s$ (inset table with elemental composition).

\section{Figure 6}

FE-TEM images of (a1-a3) ZnO-GB, and (b1-b3) Ag ${ }_{0.2} @ Z n 0,(c 1-c 3)$ Ag $_{0.4} @ Z n 0$, and (d1-d3) Ag $0.8 @ Z n 0$ $\mathrm{NCs}$, and the inset shows their corresponding SAED image.

\section{Figure 7}

FE-TEM images of (a1-a3) ZnO-GB, and (b1-b3) Ag ${ }_{0.2} @ Z n 0,(c 1-c 3)$ Ag $_{0.4} @ Z n 0$, and (d1-d3) Ag $0.8 @ Z n 0$ $\mathrm{NCs}$, and the inset shows their corresponding SAED image. 

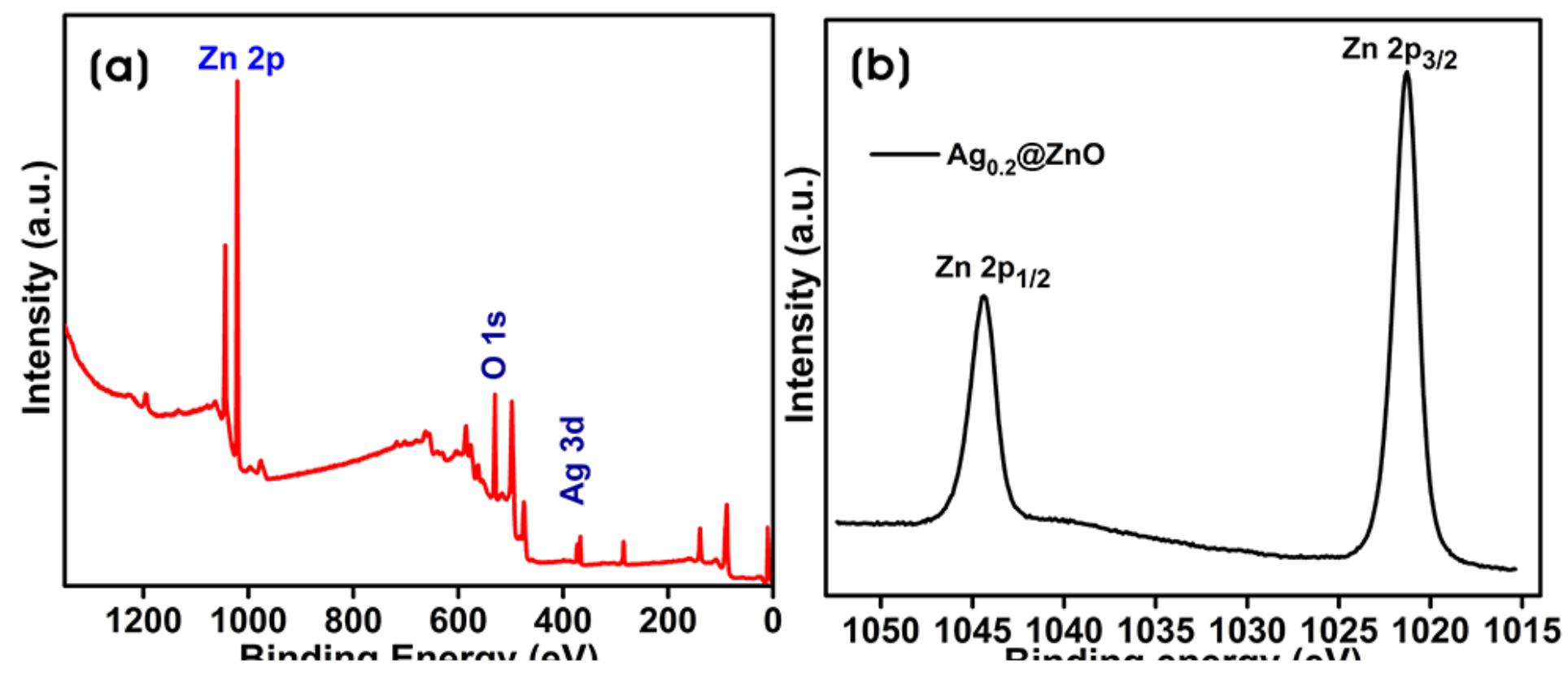

Figure 8

XPS spectrum of $\mathrm{Ag}_{0.2} @ Z n O$ NC. (a) Full survey spectrum, (b) Zn 2p spectrum, (c) deconvoluted 0 1s spectrum, and (d) Ag 3d spectrum. 

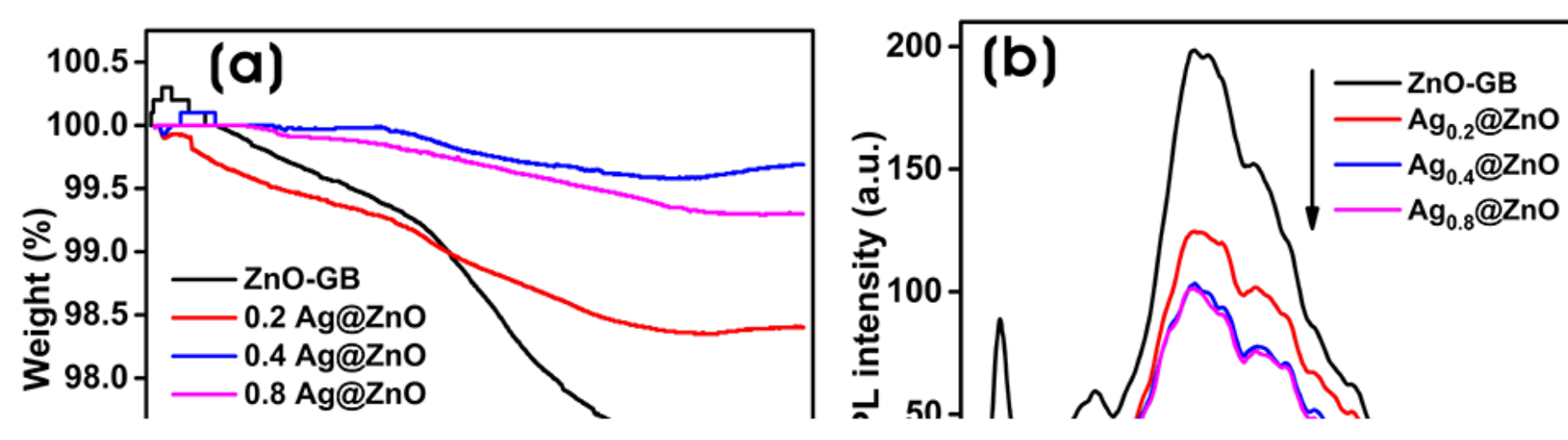

Figure 9

(a) TG analysis curve and (b) PL spectra of ZnO-GB and Ag@ZnO NCs.

Figure 10

The $\mathrm{N}_{2}$ adsorption-desorption isotherm of ZnO-GB and $\mathrm{Ag}_{0.2} @ \mathrm{ZnO} \mathrm{NC}$. (a) BET surface area analysis $\left(S_{B E T}\right)$, and (b) pore size distribution and (c) schematic diagram illustrating the photocatalytic degradation of MB and CR dyes by Ag@ZnO NCs. 

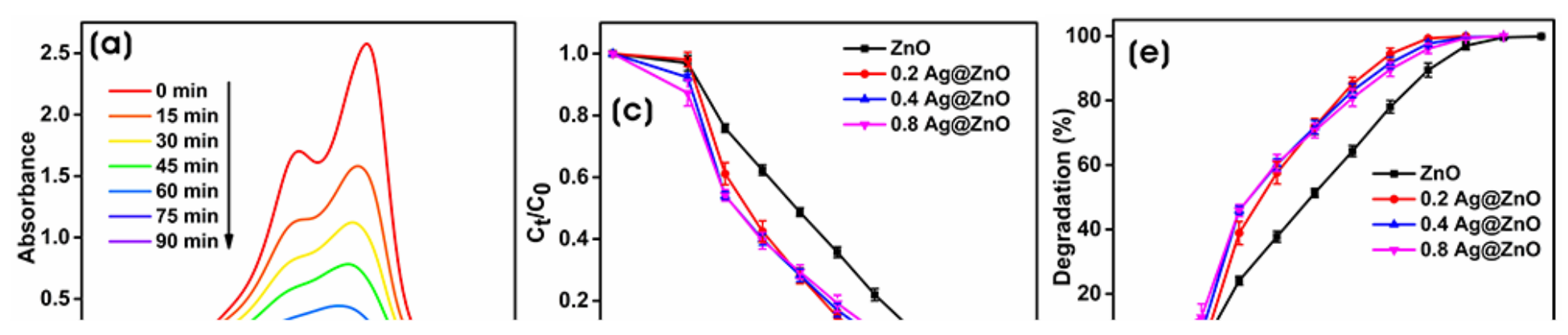

\section{Figure 11}

UV-vis absorbance spectra of the photocatalytic degradation of MB (a) and CR (b). The plot of $C_{t} / C_{0}$ against time in the degradation of MB (c) and CR (d). The degradation percentage of MB (e) and CR (f) against time by $\mathrm{Ag}_{0.2} @ \mathrm{ZnO} \mathrm{NC}$. 
Figure 12

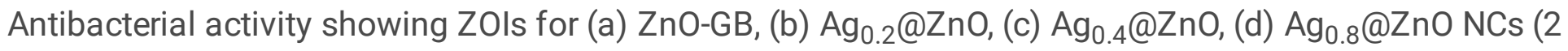
$\mathrm{mg}, 40 \mathrm{mg} / \mathrm{mL}$ ), and (e) ampicillin (positive control) against E. coli and S. aureus.

\section{Supplementary Files}

This is a list of supplementary files associated with this preprint. Click to download.

- SupplementaryMaterialScientificReports.docx 